\title{
A set on which the Łojasiewicz exponent at infinity is attained
}

\author{
by JACEK ChA̧DZYŃSKI and TADEUsz KRAsiński (Łódź)
}

\begin{abstract}
We show that for a polynomial mapping $F=\left(f_{1}, \ldots, f_{m}\right): \mathbb{C}^{n} \rightarrow \mathbb{C}^{m}$ the Lojasiewicz exponent $\mathcal{L}_{\infty}(F)$ of $F$ is attained on the set $\left\{z \in \mathbb{C}^{n}: f_{1}(z) \cdot \ldots \cdot f_{m}(z)=0\right\}$.
\end{abstract}

1. Introduction. The purpose of this paper is to prove that the Łojasiewicz exponent at infinity of a polynomial mapping $F: \mathbb{C}^{n} \rightarrow \mathbb{C}^{m}$ is attained on a proper algebraic subset of $\mathbb{C}^{n}$ defined by the components of $F$ (Thm. 1).

As a corollary we obtain a result of Z. Jelonek on testing sets for properness of polynomial mappings (Cor. 3) and a formula for the Łojasiewicz exponent at infinity of $F$ in the case $n=2, m \geq 2$, in terms of parametrizations of branches (at infinity) of zeroes of the components of $F$ (Thm. 2). This result is a generalization of the authors' result for $n=m=2$ ([CK], Main Theorem).

Before the main considerations we show some basic properties of the Łojasiewicz exponent at infinity for regular mappings, i.e. for polynomial mappings restricted to algebraic subsets of $\mathbb{C}^{n}$. We prove that the exponent is a rational number, that it is attained on a meromorphic curve (Prop. 1), and we give a condition equivalent to the properness of regular mappings (Cor. 2). These properties are analogous to ones, known in folklore, for polynomial mappings from $\mathbb{C}^{n}$ into $\mathbb{C}^{m}$. We do not pretend to the originality of proof methods; we only want to fill gaps in the literature.

The results obtained by $\mathrm{Z}$. Jelonek in $[\mathrm{J}]$ played an inspiring role in undertaking this research. On the other hand, the idea of the proof of the main theorem was taken from A. Płoski ([ $\left.\mathrm{P}_{2}\right]$, App.).

1991 Mathematics Subject Classification: Primary 14E05.

Key words and phrases: polynomial mapping, Łojasiewicz exponent.

This research was partially supported by KBN Grant No. 2 P03A 05010. 
2. The Lojasiewicz exponent. Let $F: \mathbb{C}^{n} \rightarrow \mathbb{C}^{m}, n \geq 2$, be a polynomial mapping and let $S \subset \mathbb{C}^{n}$ be an unbounded algebraic set. Put

$N(F \mid S):=\left\{\nu \in \mathbb{R}: \exists A>0, \exists B>0, \forall z \in S\left(|z|>B \Rightarrow A|z|^{\nu} \leq|F(z)|\right)\right\}$, where $|\cdot|$ is the polycylindric norm. If $S=\mathbb{C}^{n}$ we define $N(F):=N\left(F \mid \mathbb{C}^{n}\right)$.

By the Eojasiewicz exponent at infinity of $F \mid S$ we mean $\mathcal{L}_{\infty}(F \mid S):=$ $\sup N(F \mid S)$. Analogously $\mathcal{L}_{\infty}(F):=\sup N(F)$.

Before we pass to properties of the Łojasiewicz exponent we quote the known curve selection lemma at infinity (cf. [NZ], Lemma 2). We begin with a definition. A curve $\varphi:(R,+\infty) \rightarrow \mathbb{R}^{k}$ is called meromorphic at $+\infty$ if $\varphi$ is the sum of a Laurent series of the form

$$
\varphi(t)=\alpha_{p} t^{p}+\alpha_{p-1} t^{p-1}+\ldots, \quad \alpha_{i} \in \mathbb{R}^{k} .
$$

By $\|\cdot\|$ we denote the euclidian norm in $\mathbb{R}^{k}$.

Lemma 1 (Curve Selection Lemma). If $X \subset \mathbb{R}^{k}$ is an unbounded semialgebraic set, then there exists a curve $\varphi:(R,+\infty) \rightarrow \mathbb{R}^{k}$, meromorphic at $+\infty$, such that $\varphi(t) \in X$ for $t \in(R,+\infty)$ and $\|\varphi(t)\| \rightarrow \infty$ as $t \rightarrow+\infty$.

Notice that the Lojasiewicz exponent at infinity of a regular mapping $F \mid S$ does not depend on the norm in $\mathbb{C}^{n}$. So, in the rest of this section, we shall use the euclidian norm $\|\cdot\|$ in the definition of $N(F \mid S)$.

Let us introduce one more definition. A curve $\varphi=\left(\varphi_{1}, \ldots, \varphi_{m}\right):\{t \in$ $\mathbb{C}:|t|>R\} \rightarrow \mathbb{C}^{m}$ is called meromorphic at $\infty$ if $\varphi_{i}$ are meromorphic at $\infty$.

Let $F: \mathbb{C}^{n} \rightarrow \mathbb{C}^{m}, n \geq 2$, be a polynomial mapping and let $S \subset \mathbb{C}^{n}$ be an unbounded algebraic set.

Proposition 1. If \# $(F \mid S)^{-1}(0)<+\infty$, then $\mathcal{L}_{\infty}(F \mid S) \in N(F \mid S) \cap \mathbb{Q}$. Moreover, there exists a curve $\varphi:\{t \in \mathbb{C}:|t|>R\} \rightarrow \mathbb{C}^{m}$, meromorphic at $\infty$, such that $\varphi(t) \in S,\|\varphi(t)\| \rightarrow+\infty$ for $t \rightarrow \infty$ and

$$
\|F \circ \varphi(t)\| \sim\|\varphi(t)\|^{\mathcal{L}_{\infty}(F \mid S)} \quad \text { as } t \rightarrow \infty .
$$

Proof. Notice first that the set

$$
\left\{(z, w) \in S \times S:\|F(z)\|^{2} \leq\|F(w)\|^{2} \vee\|z\|^{2} \neq\|w\|^{2}\right\}
$$

is semi-algebraic in $\mathbb{C}^{n} \times \mathbb{C}^{n} \cong \mathbb{R}^{4 n}$. Then by the Tarski-Seidenberg theorem (cf. [BR], Rem. 3.8) the set

$$
\begin{aligned}
X & :=\left\{z \in S: \forall w \in S\left(\|F(z)\|^{2} \leq\|F(w)\|^{2} \vee\|z\|^{2} \neq\|w\|^{2}\right)\right\} \\
& =\left\{z \in S:\|F(z)\|=\min _{\|w\|=\|z\|}\|F(w)\|\right\}
\end{aligned}
$$

is also semi-algebraic and obviously unbounded in $\mathbb{C}^{n} \cong \mathbb{R}^{2 n}$. So, by Lemma 1 there exists a curve $\widetilde{\varphi}:(R,+\infty) \rightarrow X$, meromorphic at $+\infty$, such that $\|\widetilde{\varphi}(t)\| \rightarrow+\infty$ as $t \rightarrow+\infty$. Then there exists a positive integer $p$ such that 
$\widetilde{\varphi}$ is the sum of a Laurent series

$$
\widetilde{\varphi}(t)=\alpha_{p} t^{p}+\alpha_{p-1} t^{p-1}+\ldots, \quad \alpha_{i} \in \mathbb{C}^{n}, \alpha_{p} \neq 0 .
$$

Since $\#(F \mid S)^{-1}(0)<\infty$, there exists an integer $q$ such that $F \circ \widetilde{\varphi}$ is the sum of a Laurent series

$$
F \circ \widetilde{\varphi}(t)=\beta_{q} t^{q}+\beta_{q-1} t^{q-1}+\ldots, \quad \beta_{i} \in \mathbb{C}^{m}, \beta_{q} \neq 0 .
$$

From (2) and (3) we have

$$
\|F \circ \widetilde{\varphi}(t)\| \sim\|\widetilde{\varphi}(t)\|^{\lambda} \quad \text { as } t \rightarrow+\infty,
$$

where $\lambda:=q / p$. Let $\widetilde{\Gamma}:=\left\{z \in \mathbb{C}^{n}: z=\widetilde{\varphi}(t), t \in(R,+\infty)\right\}$. Then from (4),

$$
\|F(z)\| \sim\|z\|^{\lambda} \quad \text { as }\|z\| \rightarrow+\infty, z \in \widetilde{\Gamma} .
$$

Now, we shall show that $\mathcal{L}_{\infty}(F \mid S)=\lambda$. From (5) we have $\mathcal{L}_{\infty}(F \mid S) \leq \lambda$. Since $\widetilde{\Gamma} \subset X$ is unbounded, there exist positive constants $A, B$ such that $\|F(z)\| \geq A\|z\|^{\lambda}$ for every $z \in S$ and $\|z\|>B$. Then $\lambda \in N(F \mid S)$ and in consequence $\mathcal{L}_{\infty}(F \mid S) \geq \lambda$. Summing up, $\mathcal{L}_{\infty}(F \mid S)=\lambda \in N(F \mid S) \cap \mathbb{Q}$.

Now, we shall prove the second part of the assertion. Let $\varphi$ be an extension of $\widetilde{\varphi}$ to the complex domain, that is,

$$
\varphi(t)=\alpha_{p} t^{p}+\alpha_{p-1} t^{p-1}+\ldots,
$$

where $t \in \mathbb{C}$ and $|t|>R$. Obviously, series (6) is convergent and, as above, $\alpha_{i} \in \mathbb{C}^{n}, \alpha_{p} \neq 0$. Hence $\varphi$ is a curve, meromorphic at $\infty$, and clearly $\|\varphi(t)\| \rightarrow+\infty$ as $t \rightarrow \infty$. Moreover, $F \circ \varphi$ is an extension of $F \circ \widetilde{\varphi}$ to the complex domain and

$$
F \circ \varphi(t)=\beta_{q} t^{q}+\beta_{q-1} t^{q-1}+\ldots,
$$

where $t \in \mathbb{C}$ and $|t|>R$. Obviously, the series (7) is convergent and, as above, $\beta_{i} \in \mathbb{C}^{m}, \beta_{q} \neq 0$. From (6), (7) and the definition of $\lambda$ we get (1). Since $S$ is an algebraic subset of $\mathbb{C}^{n}$ and $\widetilde{\varphi}(t) \in S$ for $t \in(R,+\infty)$, also $\varphi(t) \in S$ for $t \in \mathbb{C},|t|>R$.

This ends the proof of the proposition.

Let $F: \mathbb{C}^{n} \rightarrow \mathbb{C}^{m}, n \geq 2$, be a polynomial mapping and $S \subset \mathbb{C}^{n}$ an algebraic unbounded set.

Directly from Proposition 1 we get

Corollary 1. $\mathcal{L}_{\infty}(F \mid S)>-\infty$ if and only if $\#(F \mid S)^{-1}(0)<+\infty$.

From Proposition 1 we also easily get

Corollary 2. The mapping $F \mid S$ is proper if and only if $\mathcal{L}_{\infty}(F \mid S)>0$.

In fact, if $\mathcal{L}_{\infty}(F \mid S)>0$, then obviously $F \mid S$ is a proper mapping. If, in turn, $\mathcal{L}_{\infty}(F \mid S) \leq 0$ then from the second part of Proposition 1 and 
Corollary 1 it follows that there exists a sequence $z_{n} \in S$ such that $\left\|z_{n}\right\| \rightarrow$ $+\infty$ and the sequence $F\left(z_{n}\right)$ is bounded. Hence $F \mid S$ is not a proper mapping in this case.

3. The main result. Now, we formulate the main result of the paper.

Theorem 1. Let $F=\left(f_{1}, \ldots, f_{m}\right): \mathbb{C}^{n} \rightarrow \mathbb{C}^{m}, n \geq 2$, be a polynomial mapping and $S:=\left\{z \in \mathbb{C}^{n}: f_{1}(z) \cdot \ldots \cdot f_{m}(z)=0\right\}$. If $S \neq \emptyset$, then

$$
\mathcal{L}_{\infty}(F)=\mathcal{L}_{\infty}(F \mid S) .
$$

The proof will be given in Section 4 .

Directly from Theorem 1 and Corollary 2 we get

Corollary 3 ([J], Cor. 6.7). If $F=\left(f_{1}, \ldots, f_{m}\right): \mathbb{C}^{n} \rightarrow \mathbb{C}^{m}, n \geq 2$, is a polynomial mapping and $S:=\left\{z \in \mathbb{C}^{n}: f_{1}(z) \cdot \ldots \cdot f_{m}(z)=0\right\}$ is not empty, then $F$ is proper if and only if $F \mid S$ is proper.

Another corollary from Theorem 1 is an effective formula for the Lojasiewicz exponent, generalizing an earlier result of the authors ([CK], Main Theorem).

Let us introduce some notions. If $\Psi:\{z \in \mathbb{C}:|z|>R\} \rightarrow \mathbb{C}^{k}$ is the sum of a Laurent series of the form

$$
\Psi(t)=\alpha_{p} t^{p}+\alpha_{p-1} t^{p-1}+\ldots, \quad \alpha_{i} \in \mathbb{C}^{k}, \alpha_{p} \neq 0
$$

then we $\operatorname{put} \operatorname{deg} \Psi:=p$. Additionally, $\operatorname{deg} \Psi:=-\infty$ if $\Psi=0$. For an algebraic curve in $\mathbb{C}^{2}$, the notions of its branches in a neighbourhood of $\infty$ and parametrizations of these branches are defined in $[\mathrm{CK}]$.

Let now $F=\left(f_{1}, \ldots, f_{m}\right): \mathbb{C}^{2} \rightarrow \mathbb{C}^{m}$ be a polynomial mapping and $S:=\left\{z \in \mathbb{C}^{2}: f_{1}(z) \cdot \ldots \cdot f_{m}(z)=0\right\}$. Assume that $S \neq \emptyset$ and $S \neq \mathbb{C}^{2}$.

THEOREM 2. If $\Gamma_{1}, \ldots, \Gamma_{s}$ are branches of the curve $S$ in a neighbourhood $Y$ of infinity and $\Phi_{i}: U_{i} \rightarrow Y, i=1, \ldots, s$, are their parametrizations, then

$$
\mathcal{L}_{\infty}(F)=\min _{i=1}^{s} \frac{\operatorname{deg} F \circ \Phi_{i}}{\operatorname{deg} \Phi_{i}} .
$$

Proof. Define $\lambda_{i}:=\operatorname{deg} F \circ \Phi_{i} / \operatorname{deg} \Phi_{i}$. If $\lambda_{i}=-\infty$ for some $i$, then (9) holds. So, assume that $\lambda_{i} \neq-\infty, i=1, \ldots, s$. Then

$$
|F(z)| \sim|z|^{\lambda_{i}} \quad \text { as }|z| \rightarrow+\infty, z \in \Gamma_{i} .
$$

Hence, taking into account the equality $S \cap Y=\Gamma_{1} \cup \ldots \cup \Gamma_{s}$ we get (9).

4. Proof of the main theorem. Let us begin with a lemma on polynomial mappings from $\mathbb{C}$ into $\mathbb{C}^{m}$. It is a generalization of a result by A. Płoski $\left(\left[\mathrm{P}_{1}\right]\right.$, Lemma 3.1) and plays a key role in the proof of the main theorem. 
Lemma 2. Let $\Phi=\left(\varphi_{1}, \ldots, \varphi_{m}\right): \mathbb{C} \rightarrow \mathbb{C}^{m}$ be a polynomial mapping and $\varphi:=\varphi_{1} \cdot \ldots \cdot \varphi_{m}$. If $\varphi$ is a polynomial of positive degree and $T$ is its set of zeroes, then for every $t \in \mathbb{C}$,

$$
|\Phi(t)| \geq 2^{-\operatorname{deg} \Phi} \min _{\tau \in T}|\Phi(\tau)| .
$$

Proof. Fix $t_{0} \in \mathbb{C}$. Let $\min _{\tau \in T}\left|t_{0}-\tau\right|$ be attained for some $\tau_{0} \in T$. If $\varphi_{i}$ is a polynomial of positive degree and has the form $\varphi_{i}(t)=c_{i} \prod_{j=1}^{\operatorname{deg} \varphi_{i}}\left(t-\tau_{i j}\right)$, then we have

$$
2\left|t_{0}-\tau_{i j}\right|=\left|t_{0}-\tau_{i j}\right|+\left|t_{0}-\tau_{i j}\right| \geq\left|t_{0}-\tau_{0}\right|+\left|t_{0}-\tau_{i j}\right| \geq\left|\tau_{0}-\tau_{i j}\right| .
$$

Hence

$$
2^{\operatorname{deg} \varphi_{i}}\left|\varphi_{i}\left(t_{0}\right)\right| \geq\left|\varphi_{i}\left(\tau_{0}\right)\right|
$$

Obviously, this inequality is also true for $\varphi_{i}$ being a constant. Since $\operatorname{deg} \Phi \geq$ $\operatorname{deg} \varphi_{i}$, from the above we get

$$
2^{\operatorname{deg} \Phi}\left|\Phi\left(t_{0}\right)\right| \geq\left|\Phi\left(\tau_{0}\right)\right| \geq \min _{\tau \in T}|\Phi(\tau)|
$$

which ends the proof.

In the sequel, $z=\left(z_{1}, \ldots, z_{n}\right) \in \mathbb{C}^{n}, n \geq 2$, and for every $i \in\{1, \ldots, n\}$ we put $z_{i}^{\prime}:=\left(z_{1}, \ldots, z_{i-1}, z_{i+1}, \ldots, z_{n}\right)$.

We state an easy lemma without proof.

LEMMA 3. Let $f: \mathbb{C}^{n} \rightarrow \mathbb{C}$ be a non-constant polynomial function and $S$ its set of zeroes. If $\operatorname{deg} f=\operatorname{deg}_{z_{i}} f$ for every $i \in\{1, \ldots, n\}$, then there exist constants $C \geq 1, D>0$ such that for every $i \in\{1, \ldots, n\}$,

$$
\left|z_{i}\right| \leq C\left|z_{i}^{\prime}\right| \quad \text { for } z \in S \text { and }\left|z_{i}^{\prime}\right|>D \text {. }
$$

Proof of Theorem 1. Without loss of generality we may assume that

(i) $S \neq \mathbb{C}^{n}$

(ii) $\#(F \mid S)^{-1}(0)<\infty$.

In fact, if (i) does not hold then (8) is obvious, whereas if (ii) does not hold then (8) follows from Corollary 1.

Obviously $N(F) \subset N(F \mid S)$. So, to prove (8) it suffices to show

$$
N(F \mid S) \subset N(F)
$$

Put $f:=f_{1} \cdot \ldots \cdot f_{m}$. From (i) we have $\operatorname{deg} f>0$. Since the sets $N(F \mid S)$ and $N(F)$ are invariant with respect to linear changes of coordinates in $\mathbb{C}^{n}$ we may assume that

$$
\operatorname{deg} f=\operatorname{deg}_{z_{i}} f, \quad i=1, \ldots, n .
$$

This obviously implies

$$
\operatorname{deg} f_{j}=\operatorname{deg}_{z_{i}} f_{j}, \quad j=1, \ldots, m, i=1, \ldots, n .
$$


It follows from (ii) and Corollary 1 that $N(F \mid S)$ is not empty. Take $\nu \in N(F \mid S)$. Then there exist $A>0, B>0$ such that

$$
|F(\zeta)| \geq A|\zeta|^{\nu} \quad \text { for } \zeta \in S,|\zeta|>B \text {. }
$$

By (11) and Lemma 3 there exist $C \geq 1, D>0$ such that for every $i \in$ $\{1, \ldots, n\}$,

$$
\left|z_{i}\right| \leq C\left|z_{i}^{\prime}\right| \quad \text { for } z \in S,\left|z_{i}^{\prime}\right|>D .
$$

Put $A_{1}:=2^{-\operatorname{deg} F} A \min \left(1, C^{\nu}\right)$ and $B_{1}:=\max (B, D)$. Take arbitrary $\check{z} \in \mathbb{C}^{n}$ such that $|\grave{z}|>B_{1}$. Clearly, $|\grave{z}|=\left|\dot{z}_{i}^{\prime}\right|$ for some $i$. Define $\varphi_{j}(t):=$ $f_{j}\left(\check{\check{z}}_{1}, \ldots, \stackrel{\circ}{z}_{i-1}, t, \stackrel{\circ}{z}_{i+1}, \ldots, \stackrel{\circ}{z}_{n}\right), \Phi:=\left(\varphi_{1}, \ldots, \varphi_{m}\right)$. Then from (12) we have

$$
\operatorname{deg} F=\operatorname{deg} \Phi .
$$

Moreover, from (11) it follows that $\varphi:=\varphi_{1} \cdot \ldots \cdot \varphi_{m}$ is a polynomial of positive degree. Then, from Lemma 2 ( $T$ is defined as in Lemma 2$)$ and (15) we have

$$
|F(\stackrel{\check{z}}{)})|=\left|\Phi\left(\check{z}_{i}\right)\right| \geq 2^{-\operatorname{deg} \Phi} \min _{\tau \in T}|\Phi(\tau)|=2^{-\operatorname{deg} F}|F(\stackrel{\circ}{\zeta})|
$$

for some $\stackrel{\circ}{\zeta}=\left(\stackrel{\circ}{z}_{1}, \ldots, \stackrel{\circ}{z}_{i-1}, \tau_{0}, \stackrel{\circ}{z}_{i+1}, \ldots, \stackrel{\circ}{z}_{n}\right), \tau_{0} \in T$. So, $\stackrel{\circ}{\zeta} \in S$. Since $|\stackrel{\gtrless}{z}|>B_{1}$ and $|\stackrel{\zeta}{\zeta}| \geq\left|\dot{z}_{i}^{\prime}\right|=|\dot{z}|$, from (16) and (13) we get

$$
|F(\stackrel{\circ}{z})| \geq 2^{-\operatorname{deg} F} A \mid \check{\zeta}^{\nu},
$$

whereas from (14),

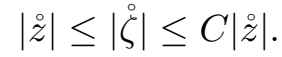

Considering two cases, when $\nu \geq 0$ and $\nu<0$, from (17) and (18) we easily get

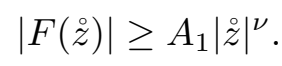

Since $\stackrel{2}{z}$ is arbitrary we have $\nu \in N(F)$.

This ends the proof of the theorem.

\section{References}

[BR] R. Benedetti and J. J. Risler, Real Algebraic and Semi-Algebraic Sets, Hermann, Paris, 1090.

[CK] J. Chądzyński and T. Krasiński, Exponent of growth of polynomial mappings of $\mathbb{C}^{2}$ into $\mathbb{C}^{2}$, in: Singularities, S. Łojasiewicz (ed.), Banach Center Publ. 20, PWN, Warszawa, 1988, 147-160.

[J] Z. Jelonek, Testing sets for properness of polynomial mappings, Inst. Math., Jagiellonian University, preprint 16 (1996), 37 pp.

[NZ] A. Némethi and A. Zaharia, Milnor fibration at infinity, Indag. Math. 3 (1992), 323-335.

$\left[\mathrm{P}_{1}\right] \quad$ A. Płoski, Newton polygons and the Eojasiewicz exponent of a holomorphic mapping of $\mathbb{C}^{2}$, Ann. Polon. Math. 51 (1990), 275-281. 
$\left[\mathrm{P}_{2}\right]$ A. Płoski, A note on the Eojasiewicz exponent at infinity, Bull. Soc. Sci. Lettres Łódź 44 (17) (1994), 11-15.

Faculty of Mathematics

University of Łódź

S. Banacha 22

90-238 Łódź, Poland

E-mail: jachadzy@imul.uni.lodz.pl

krasinsk@krysia.uni.lodz.pl 